\title{
Increases in plasma concentrations of a prostaglandin metabolite in acute airway obstruction
}

\author{
D P SKONER, P FIREMAN, H W DAVIS, ${ }^{*} \mathrm{R}$ WALL, AND L A CALIGUIRI \\ Divisions of Allergy/Immunology and ${ }^{*}$ Ambulatory Care, Department of Pediatrics, University of \\ Pittsburgh, USA
}

SUMMARY Plasma concentrations of a stable prostaglandin $F_{2} \alpha$ metabolite were measured by radioimmunoassay during and after recovery from acute airway obstruction in 15 infants. Mean (SEM) metabolite concentrations (ng/l) in plasma obtained both before (1033 (418)) and after (1470 (413)) initial treatment for airway obstruction were significantly higher than those obtained from the same subjects after resolution of the obstruction-25.5 (6.6) - and those obtained from two comparison groups. Infants positive for respiratory syncytial virus (mean 1122 (227)) had significantly higher concentrations than those who were negative (207.6 (46)). Additionally, seven subjects with a history of recurrent wheezing after resolution of airway obstruction had a significantly higher mean level $(3500(1400))$ during attacks of airway obstruction than those without $(600(100))$. These data suggest that prostaglandin $F_{2} \alpha$ mediates respiratory inflammation in airway obstruction and that trials of specific anti-inflammatory agents for the treatment of airway obstruction may be warranted.

Acute airway obstruction is commonly diagnosed in children less than 2 years of age. Potential causes include viral bronchiolitis ${ }^{1}$ and asthma, which is subsequently diagnosed in approximately $30-50 \%$ of affected infants. ${ }^{2}$ Bronchiolitis and asthma share certain signs including wheezing, hyper-reactivity of the airway, and association with viral infections; a common pathogenesis has been postulated for the two syndromes. ${ }^{3}$ Recent investigations support the hypothesis that asthma is an inflammatory disease of the lower airways. ${ }^{4-6}$ Increased concentrations of various inflammatory mediators in plasma, including histamine and neutrophil chemotactic factor of anaphylaxis, have been reported during acute asthmatic attacks ${ }^{4}$ and after bronchial provocation. ${ }^{5}$ Welliver et al reported increased concentrations of histamine and respiratory syncytial virus-specific IgE antibodies in nasopharyngeal secretions of children with respiratory syncytial virus bronchiolitis, but did not report on plasma histamine concentrations. ${ }^{7}$ Measurement of mediators in plasma may be useful as it avoids the dilutional errors common in analysis of nasal washes. Additionally, plasma concentrations might reflect the severity more accurately, as the nasal site is distant from the inflammation in the lower airways.

The purpose of the present study was to determine whether plasma concentrations of a mediator of inflammation with known bronchoconstrictive properties were increased during acute airways obstruction. Specifically, nasal and plasma concentrations of a stable prostaglandin $\mathrm{F}_{2} \alpha$ metabolite were measured in infants during and after recovery from acute airway obstruction.

\section{Patients and methods}

This research was approved by the Children's Hospital Human Experimentation Committee. Informed consent was obtained from the parents of all study subjects.

\section{STUDY DESIGN}

The criteria for study entry included age less than 1 year, history of rhinorrhea, cough and fever for no more than $\mathbf{4 8}$ hours, and airway obstruction defined as wheezing heard on auscultation of the chest associated with tachypnoea. Children with a previous history of wheezing, allergic or immunological disorders, or heart disease were excluded. Infants who fulfilled the entry criteria were enrolled in the study either after evaluation in the emergency department or following admission to hospital. Infants in the first group $(n=9)$ were studied in the outpatient clinical research centre of the Children's Hospital of Pittsburgh. Indwelling venous catheters 
were placed in six of these infants to permit collection of venous blood both before and after treatment had been started with nebulised isoetharine. Blood samples were obtained by needle venipuncture from the remaining six subjects, all of whom were studied within 48 hours of onset of symptoms having received nebulised isoetharine before enrolment. In infants with severe respiratory distress or who did not respond to the bronchodilator, arterial blood obtained for blood gas determination was also assayed for the prostaglandin metabolite. Blood was also obtained from two age matched groups for comparison.

Nasal fluids were collected as previously described ${ }^{8}$ cultured for respiratory syncytial virus in HEp2 cells, assayed for respiratory syncytial virus antigen by enzyme linked immunosorbent assay (ELISA) (Ortho Diagnostics), ${ }^{9}$ and the remainder stored frozen at $-70^{\circ} \mathrm{C}$ until assayed for prostaglandin metabolite. Nasal fluids from a subset of study subjects $(n=7)$ were assayed for protein concentration using a previously described method. ${ }^{10}$ The degree of airways obstruction was estimated clinically using the combination of a scoring system and the respiratory rate at the time of each blood collection.

Of the 15 study patients, 13 returned for reevaluation and repeat blood studies about six months after the acute attack of airway obstruction. Each parent was asked whether their child experienced any respiratory symptoms after the resolution of the acute attack. Infants in whom a reference of wheezing and tachypnoea had been documented were assigned to the group with recurrent wheezing, while the others were assigned to the group without recurrent wheezing.

\section{CLINICAL SCORE}

Each subject was evaluated by one of two investigators (DS: $n=13$, or HD: $n=2$ ). The results of a preliminary study indicated low interobserver variability in the assessment of physical signs associated with lower airways obstruction. Based on results of the examination, each subject was assigned a numerical score from 0 (least severe) to 3 (most severe) for each of the following variables: cyanosis, rib retraction, air entry, inspiratory:expiratory ratio, level of consciousness, and wheezing. The numerical values for each variable were added to yield a total score, which along with resting respiratory rate, was used to estimate severity.

\section{SUBJECTS}

Fifteen infants (mean age 3.4 months, range 1-7) were studied. A positive family history of allergies and asthma was present in nine (62\%) and $10(69 \%)$ study subjects, respectively. The duration of symptoms before enrolment into the study was two to six hours in one subject, 12-24 hours in six, and 24-48 hours in the remainder. All subjects presented with tachypnoea, ranging from 52 to 84 breaths/ minute (mean 64.4). Clinical scores on presentation ranged from 2 to 11 (mean 3.4). Of the nine subjects admitted to hospital, two were premature and required mechanical ventilation for respiratory failure. Either respiratory syncytial virus or respiratory syncytial virus antigen was detected in nasal washings of nine of $14(64 \%)$ subjects. At follow up six months after resolution of the acute airway obstruction, seven of 13 subjects $(54 \%)$ had a history of recurrent wheezing, and one wheezed on auscultation. This infant returned for a second follow up visit at which time no wheezing was detected.

For comparison, two additional groups of age matched children were studied. These comprised infants who either were normal $(n=9$, no respiratory infection) or had an upper respiratory infection, pneumonia without wheezing, or fever without obvious cause $(n=6)$.

\section{PROSTAGLANDIN METABOLITE ASSAY}

Because the half life of prostaglandin $F_{2} \alpha$ in the circulation is only a few minutes, ${ }^{11}$ concentrations of one of its stable metabolites, a 13,14-dihydro-15keto moeity, were measured using a competitive radioimmunoassay as previously described. ${ }^{4}$ The rabbit antibody used in this assay (Seragen) displayed the following cross reactivities: prostaglandin $F_{2}$ $1.7 \%$; 13,14-dihydro-15- keto- prostaglandin $\mathrm{E}_{2}$ $0 \cdot 14 \%$; 6-keto prostaglandin $F_{1} 0.02 \%$; thromboxane $B_{2} 0.02 \%$; prostaglandin $E_{2} 0.02 \%$; prostaglandin $E_{1} 0.02 \%$; 11-beta prostaglandin $F_{2} \alpha$ (Biomol) $0.02 \%$; and prostaglandin $F_{2} \beta 0.02 \%$ and 15 -ketoprostaglandin $F_{2} \beta 8.4 \%$. The sensitivity of the assay is $2.7 \mathrm{ng} / 1$ and samples with lower concentrations of metabolite are reported as $<2.7 \mathrm{ng} / \mathrm{l}$. Plasma prostaglandin metabolite concentrations are expressed as $\mathrm{ng} / \mathrm{l}$; concentrations in nasal fluid were analysed as both $\mathrm{ng} / \mathrm{l}$ and $\mathrm{ng} / \mathrm{g}$ of protein for normalisation.

\section{DATA ANALYSIS}

Samples obtained from study subjects were grouped by collection time, site of collection (artery, vein, nose), respiratory syncytial virus positivity, and recurrence of wheezing. The mean plasma and nasal prostaglandin metabolite concentrations of the groups were calculated and compared. Indicators of severity (respiratory rate and clinical score) and family histories (asthma and allergies) were also correlated with corresponding concentrations of metabolite. Unless otherwise specified, data are 
presented as mean (SEM) and were analysed by Student's $t$ test. ${ }^{12}$

Subjects' blood samples were divided into three groups for analysis: group I-samples collected on presentation to the emergency department before treatment for acute airway obstruction $(n=9)$; group II-samples collected after treatment had been started, but within 48 hours of the onset of symptoms $(n=19)$; and group III-samples collected at the follow up visit, when the infant was free of symptoms $(n=13)$. Normal infants with no respiratory infection $(n=9)$ and infants with upper respiratory infections, pneumonia without wheezing, or fever without obvious cause $(n=6)$ comprised groups IV and V, respectively.

\section{Results}

Prostaglandin metabolite values before and after initial treatment and after resolution ranged from 74-3600 ng/l, 13-7240 ng/l, and <2.7-84 ng/l, respectively. Concentrations from both before (1033 (418)) and after (1470 (413)) treatment had been started were significantly higher $(p<0 \cdot 005)$ than those from the same subjects after resolution of symptoms $(25.5(6 \cdot 6))$. The difference between concentrations before and after treatment was not significant and the value after resolution was not significantly different from that of normal infants $(42.0(20.4))$, or infants in the comparison group (33.5 (17)). These latter values do not differ from those reported for normal individuals by radioimmunoassay, ${ }^{13}$ gas chromatography and mass spectrometry, ${ }^{14} 15$ and with the calculated rate of prostaglandin $F_{2} \alpha$ synthesis in humans. ${ }^{15}$ The plasma prostaglandin metabolite concentration of the single infant in the comparison group (group V) who had pneumonia without wheezing was less than the lowest detectable concentration. To illustrate the group differences, plasma concentrations of the metabolite were converted to log values (fig 1 ). The mean (SD) log values of groups $I$ to $V$ inclusive were $2.7(0.53), 2.8(0.73), 1.19(0.47)$, $1.21(0.64)$ and $0.6(1.2)$, respectively.

Prostaglandin metabolite concentrations in the subgroups of infants who were artificially ventilated or who had intravenous catheters were not significantly different from those in the other study subjects. This suggests that the increases in plasma concentrations were not iatrogenically induced by manoeuvres undertaken in the care of the patients. In support of this hypothesis, intravenous catheters were inserted in three healthy individuals, and did not result in increases in plasma prostaglandin metabolite concentrations 5,15 , and 60 minutes later (unpublished observations).

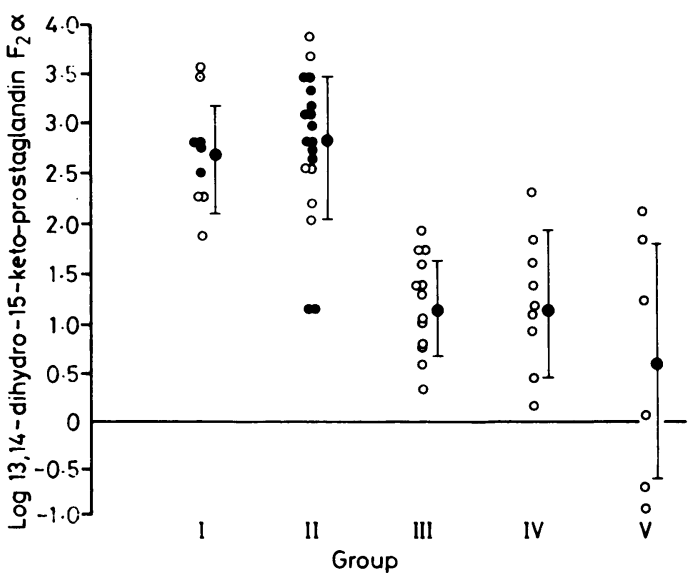

Fig 1 Log distribution of plasma concentrations of 13 , 14-dihydro-15-keto-prostaglandin $F_{2} \alpha$ in subjects with airway obstruction (groups I, II, and III) and in comparison subjects (groups IV and V). Vertical lines indicate mean $(S D)$. Closed circles indicate subjects positive for respiratory syncytial virus. Airway obstruction samples are grouped by collection time: group I-before treatment for acute airway obstruction ( $n=9)$; group II-after initial treatment, but within 48 hours of onset of symptoms $(n=19)$; and group III-after resolution of acute airway obstruction ( $n=13)$. Group IV-normal children $(n=9)$; group $V$-infants with upper respiratory infection, pneumonia without wheezing or fever without obvious cause $(n=6)$.

More than one blood sample was obtained from six of the subjects during the study period. As shown in fig 2 , three representative subjects had increased, decreased, or unchanged plasma prostaglandin metabolite concentrations after bronchodilator treatment had been started. All values returned to normal at the follow up visit, except the one patient who was wheezing at the six month follow up visit. This patient was not wheezing when evaluated one month later, at which time the concentration had returned to normal $(6 \cdot 2 \mathrm{ng} / \mathrm{l})$.

The concentrations (ng/l) in arterial blood obtained after treatment $(1782(432.9), n=5)$ were higher than those in venous blood taken after treatment from either the same subjects $(952.4(136 \cdot 2))$ or from the whole group $(1470(413 \cdot 2), n=19)$, but these differences were not significant. This arterial venous difference was also observed when simultaneous venous $(939.2 \mathrm{ng} / \mathrm{l})$ and arterial $(2921 \mathrm{ng} / \mathrm{l})$ samples were obtained from one subject who required artificial ventilation for respiratory failure.

Prostaglandin metabolite concentrations in nasal secretions $(\mathrm{n}=12)$ ranged from $<2.7$ to $1645 \mathrm{ng} / \mathrm{l}$, mean $383.8(120 \cdot 6) \mathrm{ng} / \mathrm{l}$. After normalisation with 


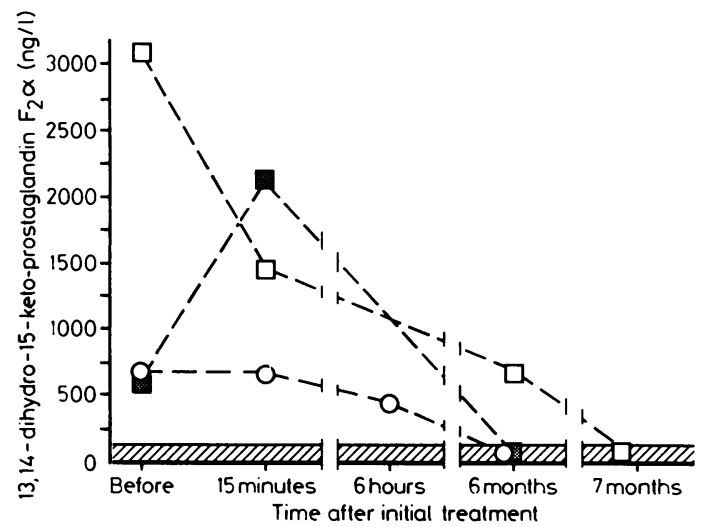

Fig 2 Patterns of change in plasma 13,14-dihydro-15-ketoprostaglandin $F_{2} \alpha$ concentrations (ng/l) before and after initial treatment for acute airway obstruction in three typical infants. Note persistently increased concentration in one infant (open squares) at six month follow up visit when wheezing, then return to normal range at seven month follow up visit when not wheezing. Cross hatched area represents two standard deviations above the mean for normal age matched infants ( $95 \%$ confidence interval).

protein content, nasal prostaglandin metabolite concentrations ranged from 172 to $8225 \mathrm{ng} / \mathrm{g}$ protein, mean 1781 (1088). The increases were often accompanied by similar increases in plasma concentrations, but a significant positive correlation between these two variables could not be shown before or after normalisation (Pearson product moment correlation coefficient $).{ }^{16}$

Plasma and nasal concentrations of prostaglandin metabolite were compared with corresponding indicators of severity (clinical score and respiratory rate) for each subject. Even though both plasma and nasal metabolite concentrations were often considerably raised in the most severely ill infants, a positive correlation could not be demonstrated for any of the clinical variables using the Spearman rank correlation coefficient. ${ }^{17}$ Necessity for hospital admission was also used as an indicator of more severely ill infants, and metabolite concentrations in infants in hospital (1147 (656)) were not significantly different from outpatients (1087 (403)).

Nasal and plasma metabolite concentrations during acute attacks of airways obstruction were compared for infants with and without recurrent wheezing after resolution. Concentrations both before and after treatment were higher in the subjects with recurrent wheezing than in those without, but this difference was significant $(p<0.025)$ only for the concentrations after treatment (3500 (1400) ng/l compared with $600(100) \mathrm{ng} / \mathrm{l}$, respectively). Nasal metabolite concentrations were not significantly different between the two groups.

When subjects were grouped according to the presence or absence of respiratory syncytial virus or respiratory syncytial virus antigen in nasal washings, infants positive for respiratory syncytial virus $(1122(226.7) \mathrm{ng} / \mathrm{l})$ had significantly higher plasma metabolite concentrations $(p<0.05)$ than those who were negative for respiratory syncytial virus $(207 \cdot 6$ $(46 \cdot 2) \mathrm{ng} / \mathrm{l})$ during acute attacks of airway obstruction (fig 1). A significant difference between the two groups could not, however, be shown for nasal concentrations. Even though plasma concentrations were higher in infants with family histories of allergies and asthma (1468 (523) and 1386 (496), respectively) compared with those without such histories (688 (440) and 794 (514), respectively), the differences were not significant. Additionally, metabolite concentrations were not significantly different in infants whose symptoms had lasted 12 to 24 hours (1854 (707)) compared with those whose symptoms had lasted 24 to 48 hours (571 (421)).

\section{Discussion}

The results of this study show for the first time that plasma levels of a prostaglandin $F_{2} \alpha$ metabolite are considerably increased (fifty fold) in infants with acute airway obstruction, and return to normal after the acute process has resolved. Several pieces of evidence suggest that many of these infants had bronchiolitis, but whether this represented their first episode of asthma is not clear. Lesser increases of the same metabolite were observed during acute asthmatic attacks by our laboratory staff, ${ }^{4}$ and during provoked episodes of wheezing and bronchospasm by others. ${ }^{14}$ These increases in plasma concentrations probably reflect much higher local concentrations of the metabolite's active precursor molecule, prostaglandin $F_{2} \alpha$, in the tissue of origin. Prostaglandin $F_{2} \alpha$ is a potent bronchoconstrictor ${ }^{18}$ and secretogogue, ${ }^{19}$ the primary synthetic and metabolic site of which is the lung. ${ }^{13}$ These observations suggest that prostaglandin $F_{2} \alpha$ is a mediator of airway inflammation in airway obstructive disorders.

The likely tissue source for the prostaglandin metabolite is the lung, which is a major site for prostaglandin synthesis by the cyclo-oxygenase pathway of arachidonic acid metabolism, and for release and degradation of prostaglandins. ${ }^{13}$ Carrier mediated prostaglandin uptake by lung tissue, followed by cytoplasmic enzyme inactivation and subsequent release of 15-keto and 13,14-dihydro-15- 
keto-metabolites ${ }^{20}$ has been postulated. Thus metabolites appear in effluent pulmonary venous and systemic arterial blood, capillary vascular beds and then venous plasma. The arteriovenous gradient for the prostaglandin metabolite observed in the present study suggests that a portion of the circulating metabolite is removed or degraded in the capillary microvascular bed.

Both pulmonary macrophages ${ }^{21}$ and epithelial cells $^{22}$ have been reported to synthesise prostaglandins. Cellular prostaglandin synthesis can be triggered by diverse physiological and pathological stimuli, including viruses. Respiratory syncytial virus and other viral agents could directly or indirectly induce prostaglandin synthesis. A pathogenetic role for IgE dependent hypersensitivity has been postulated in bronchiolitis. ${ }^{7}$ A virus specific, type I hypersensitivity reaction with histamine release could result in the direct stimulation of pulmonary prostaglandin synthesis and release in airway obstruction. Alternatively, viruses may stimulate prostaglandin synthesis indirectly. Interferon, a glycoprotein secreted by cells in response to viral infection, is a potent inducer of cellular prostaglandin synthesis and secretion. ${ }^{23}$ In support of the hypothesis of a virus induced prostaglandin synthesis, plasma metabolite concentrations were significantly higher $(\mathrm{p}<0.05)$ in infants with respiratory syncytial virus positive airway obstruction when compared with those who were respiratory syncytial virus negative.

In contrast to results reported by Welliver $e$ t al for histamine ${ }^{7}$ nasal prostaglandin metabolite concentrations did not correlate with two clinical indicators of severity, even after normalisation to protein content. Although the reason for this discrepancy is unclear, several possible explanations should be considered. The nasal mucosa may not synthesise large quantities of prostaglandin $F_{2} \alpha$ during airway obstruction or, alternatively, could lack the enzymes necessary for conversion of prostaglandin $F_{2} \alpha$ to its stable metabolite. The concentrations measured in nasal fluids could also partly represent plasma exudation of metabolite formed in the lung. A second possible explanation is the variability inherent in the technique of nasal fluid collection. The volume of fluid recovered during washing procedures is variable and could result in inconsistent dilutions and spurious metabolite concentrations. A lack of correlation after normalisation of metabolite to protein concentrations, however, makes this possibility unlikely. Alternatively and even less likely, the indicators used in the present study may lack sufficient sensitivity or specificity in identifying severely ill infants. A similar lack of correlation between clinical severity and plasma metabolite concentrations supports this hypothesis.

Even though the incidence of recurrent wheezing in this study is similar to that reported previously, the follow up period is probably too short to detect all subjects who will ultimately have recurrent wheezing. Indeed, wheezing may not recur until several years after the acute attack of airway obstruction. Further prospective studies of this group are being conducted to clarify this issue. None the less, in the present study, infants who carried on wheezing after resolution of airway obstruction had significantly higher plasma metabolite concentrations during the acute illness than those who did not. This suggests that an increased pulmonary concentration of prostaglandin $F_{2} \alpha$ predisposes infants to airway hyper-reactivity and recurrences of wheezing or, alternatively, that a relative hyper-responsiveness of prostaglandin synthesising cells is present in the subgroup of infants who later experience recurrences.

The elaboration of prostaglandin $F_{2} \alpha$ and perhaps other bronchoconstrictive mediators of inflammation during acute airway obstruction should provide a stimulus for the development of specific antiinflammatory agents to supplement bronchodilators in the treatment of infantile airway obstruction.

The authors acknowledge the technical assistance of Jacqueline Suttner, RN, BSN, the editorial assistance of WJ Doyle, PhD, the assistance of EP Tanner, MS in data analysis, and the cooperation of the emergency department nursing staff of the Children's Hospital of Pittsburgh. This work was supported in part by NIH grant number 3 M01 RR00084-23S1 (to Dr Skoner), United Way Health Research and Services Foundation BB-67, and the American Lung Association of Pennsylvania. The prostaglandins were generously supplied by Dr F Fitzpatrick of Upjohn.

\section{References}

${ }^{1}$ Henderson FW, Clyde WA, Collier AM, Denny FW. The etiologic and epidemiologic spectrum of bronchiolitis in pediatric practice. J Pediatr 1979;95:183-90.

2 Rooney JC, Williams HE. The relationship between proven viral bronchiolitis and subsequent wheezing. J Pediatr 1971;79: 744-7.

${ }^{3}$ McIntosh K. Bronchiolitis and asthma: possible common pathogenetic pathways. J Allergy Clin Immunol 1976;57:595-604.

4 Skoner DP, Page R, Asman B, Gillen L, Fireman P. Plasma elevations of histamine and a prostaglandin metabolite in acute asthma. Am Rev Respir Dis 1988;137:1009-14.

${ }^{5}$ Lee TH, Nagakura T, Cromwell O, Brown MJ, Causon R, Kay AB. Neutrophil chemotactic activity and histamine in atopic and nonatopic subjects after exercise-induced asthma. Am Rev Respir Dis 1984;129:409-12.

6 DeMonchy JG, Kauffman HF, Venge P, et al. Bronchoalveolar eosinophilia during allergen-induced late asthmatic reactions. Am Rev Respir Dis 1985;131:373-6.

7 Welliver RC, Wong DT, Sun M, Middleton E, Vaughan RS Ogra PL. The development of respiratory syncytial virusspecific IgE and the release of histamine in nasopharyngeal secretions after infection. $N$ Engl J Med 1981;305:841-6.

8 Hall CB, Douglas RG. Clinically useful method for the isolation of respiratory syncytial virus. J Infect Dis 1975;131:1-5. 
${ }^{9}$ Lauer BA, Masters HA, Wren CG, Levin MJ. Rapid detection of respiratory syncytial virus in nasopharyngeal secretions by enzyme-linked immunosorbent assay (ELISA). J Clin Microbiol 1985;22:782-5.

10 Lowry OH, Rosebrough NJ, Farr AL, Randall RJ. Protein measurement with the Folin phenol reagent. $J$ Biol Chem 1951;193:265-75.

${ }^{11}$ Ferreira SH, Vane JR. Prostaglandins: their disappearance from and release into the circulation. Nature 1967;216:868-73.

12 Snedecor GW, Cochran WG. The normal distribution. In: Snedecor GW, Cochran WG, eds. Statistical methods. 7th ed. Ames: Iowa State University Press, 1980:54.

13 Robinson C, Hardy CC, Holgate ST. Pulmonary synthesis, release and metabolism of prostaglandins. J Allergy Clin Immunol 1985;76:265-71.

14 Green K, Hedqvist P, Svanborg N. Increased plasma levels of 15-keto-13,14-dihydro-prostaglandin $F_{2} \alpha$ after allergenprovoked asthma in man. Lancet 1974;ii:1419-21.

15 Samuelsson B, Green K. Endogenous levels of 15-keto-dihydroprostaglandins in human plasma. Biochemical Medicine 1974;11: 298-303.

16 Snedecor GW, Cochran WG. The normal distribution. In: Snedecor GW, Cochran WG, eds. Statistical methods. 7th ed. Ames: Iowa State University Press, 1980:175.
17 Siegel S. Nonparametric statistics for the behavioural sciences. Tokyo: McGraw-Hill, Kogakusha, 1956:202-13.

${ }^{18}$ Demers LM. Prostaglandins. In: Race G, ed. Laboratory medicine. Hagerstown: Harper and Rowe, 1983:1-21.

19 Marom Z, Shelhamer JH, Kaliner M. The effects of arachidonic acid derivatives on the release of mucous glycoproteins from human airways. J Clin Invest 1981;67:1695-1702.

${ }^{20}$ Robinson C, Hoult JRS. Inactivation of prostaglandins in the perfused rat lung. Biochem Pharmacol 1982;31:633-8.

21 Kouzan S, Brody AR, Nettesheim P, Eling T. Production of arachidonic acid metabolites by macrophages exposed in vitro to asbestos, carbonyl iron particles, or calcium ionophore. Am Rev Respir Dis 1985;131:624-32.

22 Xu Gl, Sivarajah K, Wu R, Nettesheim P, Eling T. Biosynthesis of prostaglandins by isolated and cultured airway epithelial cells. Exp Lung Res 1986;10:101-14.

${ }^{23}$ Fitzpatrick FA, Stringfellow DA. Virus and interferon effects on cellular prostaglandin biosynthesis. J Immunol 1980;125:431-7.

Correspondence to Dr DP Skoner, Children's Hospital of Pittsburgh, 125 DeSoto Street, Pittsburgh, PA 15213, USA.

Accepted 13 February 1989 